\title{
Aggressive undifferentiated pleomorphic sarcoma of the stomach involving long-term survival: A case report and literature review
}

\author{
YOHEI OGURI ${ }^{1}$, HARUHIKO CHO ${ }^{1}$, RYOUKI OOHINATA ${ }^{1}$, HARUNA ONOYAMA ${ }^{1}$, \\ RYOSUKE TAKADA $^{1}$ and TORU MOTOI ${ }^{2}$
}

Departments of ${ }^{1}$ Surgery and ${ }^{2}$ Pathology, Tokyo Metropolitan Cancer and Infectious

Disease Center Komagome Hospital, Tokyo 113-0021, Japan

Received June 7, 2018; Accepted September 17, 2018

DOI: $10.3892 /$ mco.2018.1739

\begin{abstract}
Primary gastric undifferentiated pleomorphic sarcoma (UPS) is a rare disease with insufficient long-term follow-up data. In the present study, a 70-year-old male complained of abdominal fullness and visited our hospital. Abdominal computed tomography revealed a large tumor in the upper part of the stomach, which was accompanied by smaller tumors in the small intestinal mesentery. An endoscopic ultrasound-guided fine-needle biopsy examination of the gastric tumor revealed features of pleomorphic sarcoma and high-grade spindle-shaped cells. Total gastrectomy was performed on the primary tumor, together with combined resection of the small intestine for the metastatic tumors. However, the tumor recurred in the mesentery of the sigmoid colon 6 months after the operation. A second operation was performed to resect the recurrent tumor. Since the second surgical procedure, the patient has remained free from recurrence for $>7$ years. Although the prognosis of abdominal UPS was considered to be poor, even after curative surgery, the present case experienced a long-term survival of gastric UPS after undergoing surgical resection alone.
\end{abstract}

\section{Introduction}

Undifferentiated pleomorphic sarcoma (UPS), which was formerly known as malignant fibrous histiocytoma, is a high-grade sarcoma, which mainly arises from the soft tissue of

Correspondence to: Dr Haruhiko Cho, Department of Surgery, Tokyo Metropolitan Cancer and Infectious Disease Center Komagome Hospital, 3-18-22 Honkomagome, Bunkyo-ku, Tokyo 113-0021, Japan

E-mail: choharuhiko@cick.jp

Abbreviations: UPS, undifferentiated pleomorphic sarcoma; CT, computed tomography; MRI, magnetic resonance imaging; EUS, endoscopic ultrasound; FNB, fine-needle biopsy; MDM2, mouse double minute 2 homolog; CDK4, cyclin-dependent kinase 4; GIST, gastrointestinal stromal tumors; DLPS, dedifferentiated liposarcoma

Key words: gastric sarcoma, undifferentiated pleomorphic sarcoma, metastasis the extremities and can appear at any age, but typically presents in the 5th-7th decades of life and exhibits a slight male predominance (1). Histopathologically, it is characterized by pleomorphic and spindle-shaped tumor cells and a storiform growth pattern; however, it lacks a definitive line of differentiation (2). To date, several cases of UPS that arose in the gastrointestinal tract have been reported, but these reports did not include long-term follow-up data. We report a case of UPS, in which the patient survived for more than 7 years after the surgical resection of the primary gastric tumor and metachronous metastases.

\section{Case presentation}

A 70-year-old male visited his primary care doctor, complaining of abdominal fullness and a palpable large mass in his lower abdomen. He was referred to our hospital for further investigation. He had a history of hypertension and had undergone resection of the right upper pulmonary lobe for tuberculosis 50 years ago. His blood examination findings only showed an elevated C-reactive protein level. An abdominal computed tomography (CT) scan revealed a $14-\mathrm{cm}$ oval tumor in the wall of the stomach and two $10-\mathrm{cm}$ tumors in the mesentery of the small intestine (Fig. 1). During magnetic resonance imaging (MRI), the tumor exhibited low signal intensity on T1-weighted images and high and diffuse signal intensity on T2-weighted images (Fig. 2). It also demonstrated a diffusional constraint on diffusion-weighted images. Upper gastrointestinal endoscopy showed a submucosal tumor, and endoscopic ultrasound (EUS) revealed a hypoechoic solid tumor, which was contiguous with the proper muscular layer (Fig. 3). An endoscopic ultrasound-guided fine-needle biopsy (EUS-FNB) was performed, and the specimen was found to contain atypical cells with pleomorphic or bizarre nuclei and a necrotic background, which was consistent with high-grade sarcoma. Atypical cells showed no definite differentiation by immunohistochemically, which were only positive for vimentin, and negative for $\alpha$-smooth muscle actin, cytokeratin (AE1/AE3), epithelial membrane antigen, desmin, S-100 protein, c-Kit, CD34 and Melan-A. Based on these findings, we decided to perform total gastrectomy combined with resection of the accompanying tumors because it was considered that systemic therapy would not be effective. Twelve days after the EUS-FNB examination, the patient underwent total 
gastrectomy and combined resection of the small intestinal tumors. An intraoperative examination confirmed that the largest tumor originated from the anterior wall of the upper stomach and occupied the space between the stomach and the transverse colon, whereas the other tumors located in the ileal mesentery. There were no other masses in the abdominal cavity. Intraoperative peritoneal washing cytology was negative for tumor cells. As none of the tumors had invaded the adjacent organs, they were wholly resected via total gastrectomy and partial resection of the ileum. The gastric tumor was resected en bloc combined with D2 lymphadenectomy according to the Japanese Classification of Gastric Carcinoma guidelines (3).

Macroscopically, the resected gastric tumor was a well-demarcated solid grayish-white tumor, measuring $14 \times 12 \times 10 \mathrm{~cm}$ in size, which displayed extensive central necrosis and peripheral lobulation and protruded into the abdominal cavity (Fig. 4A and B). The other extra-gastric tumors measured $12.5 \times 11 \times 8.5$ and $11.5 \times 9.5 \times 9 \mathrm{~cm}$ in size, respectively. Microscopically, the tumors were composed of atypical spindle-shaped and pleomorphic cells with large pleomorphic or bizarre nuclei (Fig. 4C). Mitotic figures, including abnormal mitoses, were frequently encountered (5-6/high-power field). The tumor displayed expansive growth without infiltrating into the adjacent tissue. No vascular invasion or regional lymph node metastasis was observed (0/44). Immunohistochemically, the atypical cells were positive for vimentin, but negative for cytokeratin (AE1/AE3 and CAM 5.2), epithelial membrane antigen, desmin, S-100 protein, c-Kit, CD34, mouse double minute 2 homolog (MDM2), cyclin-dependent kinase 4 (CDK4), and melan-A. The final pathological diagnosis was UPS of the stomach with peritoneal dissemination. The patient's postoperative course was uneventful; however, 6 months later, follow-up CT showed a tumor located in the ligament of the sigmoid colon, and so partial resection of the sigmoid colon was performed. The histological and immunohistochemical features of the resected tumor were almost the same as those of the primary tumor. Peritoneal washing cytology was positive for atypical cells. No adjuvant chemotherapy was administered because there is no standard chemotherapy regimen for abdominal UPS, UPS exhibits a low chemotherapy response rate, and chemotherapy can have side effects. Seven years after the surgery, the patient remains tumor-free.

\section{Discussion}

Gastric mesenchymal tumors are much rarer than gastric epithelial tumors. However, gastric mesenchymal tumors include both benign and malignant tumors. Among them, gastrointestinal stromal tumors (GIST) are the most common type of mesenchymal tumor, and most GIST exhibit benign behavior so there is little difficulty with their pathological diagnosis. Conversely, gastric sarcomas are reported to account for $<1-3 \%$ of all gastric tumors, and they are difficult to diagnose based on their clinical and pathological findings (4). UPS is the commonest type of high-grade malignant sarcoma found in elderly people and most are asymptomatic but some case was reported fever as chief complaint because UPS producing activity cytokines as G-CSF (5). It is characterized as a pleomorphic sarcoma involving spindle-shaped cells, which is not very disease-specific, and does not exhibit marked

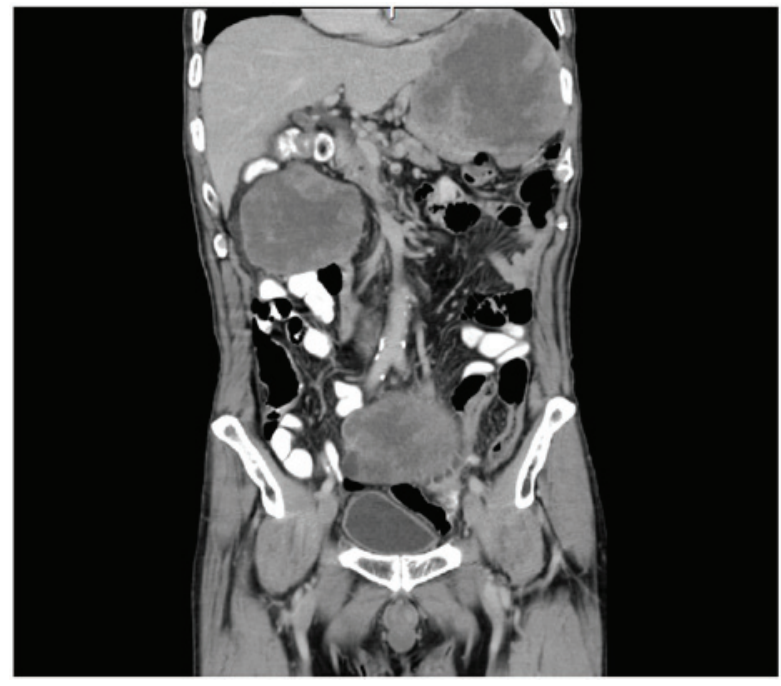

Figure 1. Contrast-enhanced abdominal computerized tomography revealed a $14-\mathrm{cm}$ oval tumor in the stomach and $10-\mathrm{cm}$ tumors in the mesentery of the small intestine. The tumor in the stomach was encapsulated and contained a central low-density region.

cellular differentiation. Therefore, it is essentially diagnosed by exclusion, so it is necessary to carefully exclude other sarcomas whose histology overlap with that of UPS using electron microscopy and immunohistochemical techniques.

Regarding primary sarcoma of gastric origin, the main differential diagnoses for such cases include leiomyosarcoma and malignant peripheral nerve sheath tumors. However, in the present case these tumors were excluded because of the lack of neurogenic marker and smooth muscle marker expression $(6,7)$. In addition, a rare type of malignant GIST, dedifferentiated GIST, in which a high-grade malignant sarcoma component is found in an ordinary GIST, was also considered; however, it was excluded due to the lack of a typical GIST component and the fact that the tumor was completely negative for c-kit (8). In this case, multiple tumors were detected in the abdominal cavity, including both simultaneous and metachronous tumors. Therefore, we also had to consider dedifferentiated liposarcoma (DLPS), which is a more common type of sarcoma of intra-abdominal/retroperitoneal origin that can involve the stomach and produce multiple tumor nodules. Although the histology of the dedifferentiated component of DLPS is indistinguishable from that of UPS, it typically contains a well-differentiated component, which exhibits marked adipocytic differentiation. Cytogenetically, DLPS is characterized by gene amplification of the $12 \mathrm{q} 13-15$ region, resulting in the overexpression of MDM2 and CDK4 (9). In the current case, DLPS was excluded because the tumor lacked adipocytic differentiation and did not overexpress MDM2 or CDK4.

Although there are no CT findings specific to UPS as far as we know, previous review of peritoneal sarcomatosis reported that peritoneal implants and mesenteric involvement were well-defined, and neither diffuse thickening nor calcifications (10). Also in our case, tumors were found to have well-defined shapes and central hypo-density areas as common characteristics of peritoneal sarcomas. The other report indicated the apparent diffusion coefficient (ADC) values caluculated from diffusion-weighted MRI was independent 

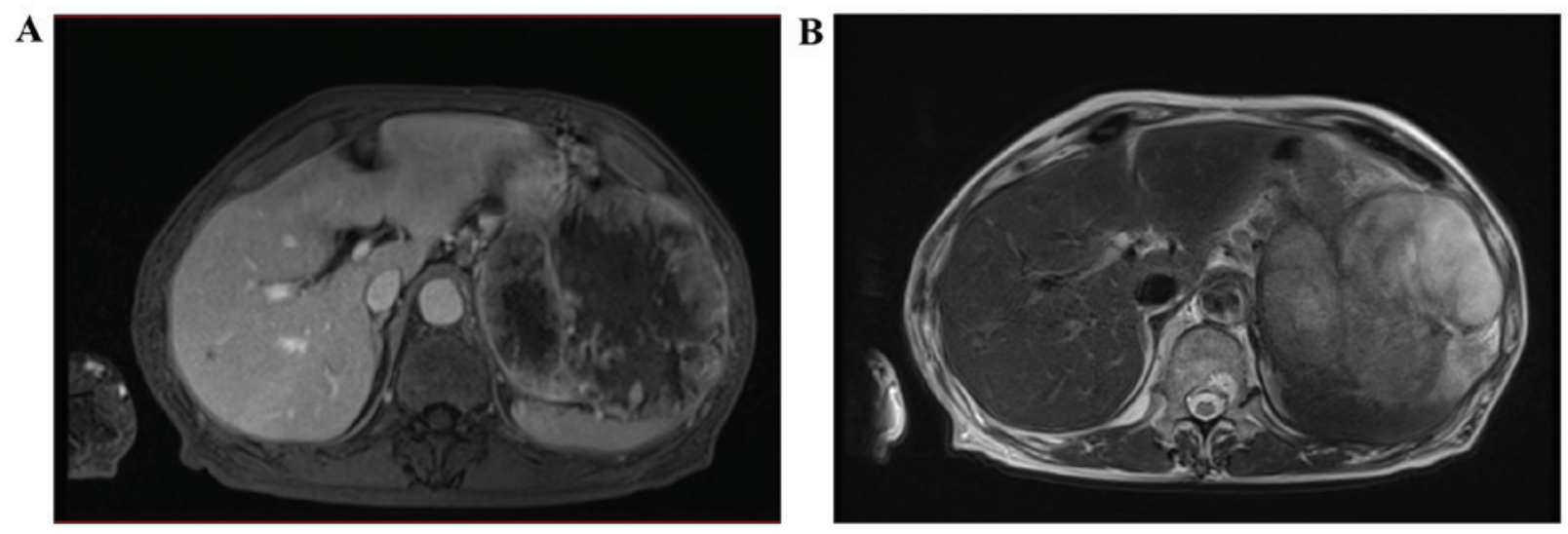

Figure 2. Magnetic resonance imaging revealed that the tumor exhibited low signal intensity on T1-weighted images and high signal intensity on T2-weighted images. (A) T1-weighted image, (B) T2-weighted image.
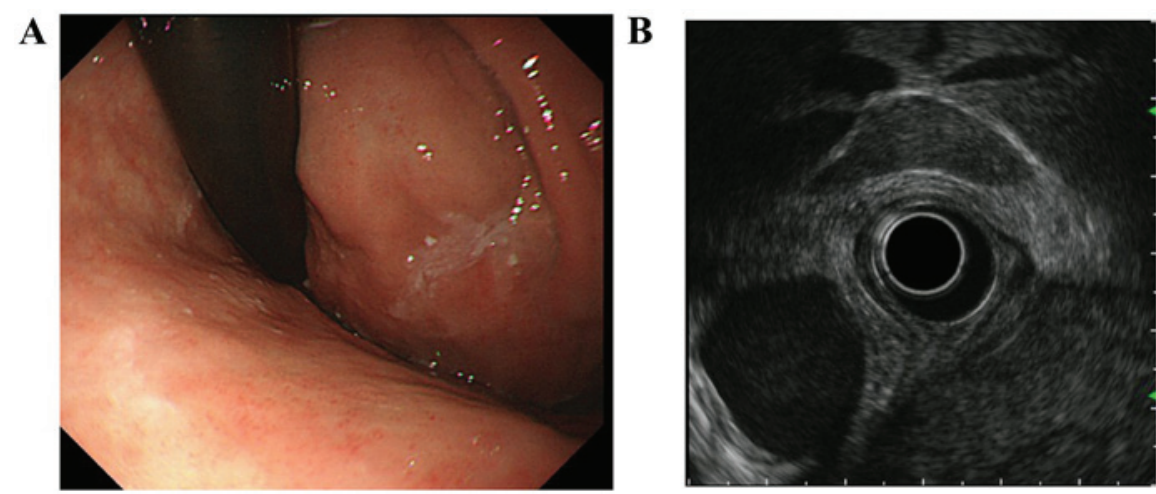

Figure 3. Upper gastrointestinal endoscopy revealed a submucosal tumor in the cardia of the stomach. EUS showed a hypoechoic mass originating from the submucosa. (A) Upper gastrointestinal endoscopic imaging, (B) EUS imaging.
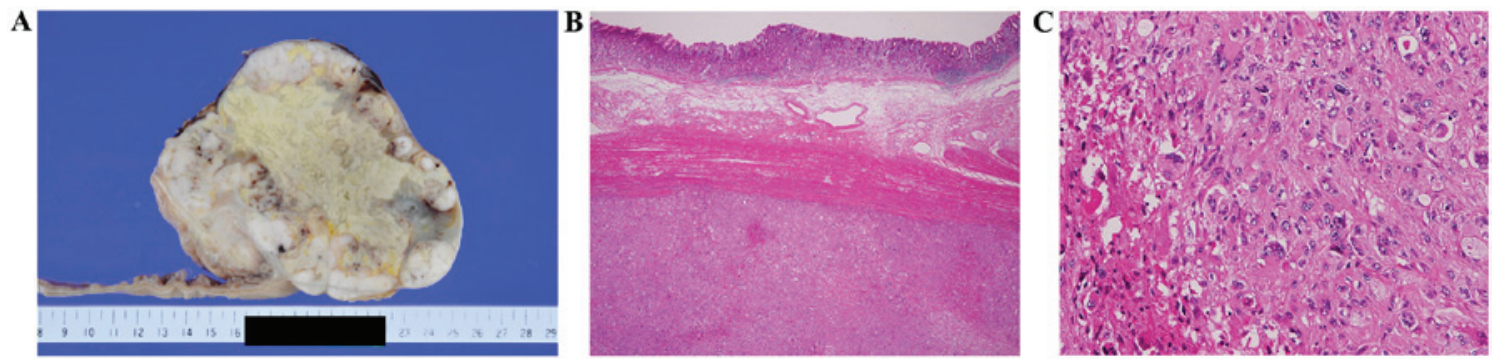

Figure 4. (A) Gross findings of the resected gastric specimen. The tumor was solid and lobulated and necrosis was evident in the center of the tumor. (B) Gross findings of the resected gastric specimen. The tumor arose from the submucosal layer. (C) The tumors were composed of atypical irregularly arranged spindle-shaped cells with large pleomorphic or bizarre nuclei.

prognostic factor in gastric cancer (11). We might be able to apply ADC value of gastric UPS for prognostic indication.

With regard to the evaluation of the tumor's histological grade, the UPS was a large undifferentiated tumor containing frequent mitoses and extensive necrosis. According to the French Federation of Comprehensive Cancer Centers grading system for soft tissue sarcomas, it exhibited a tumor differentiation score of 3 , a mitotic count score of 3 , and a necrosis score of 2 and had an overall classification of grade 3 , which is the highest grade (12). It is worth specifically mentioning that the patient in this case demonstrated an unexpectedly good prognosis in spite of the tumor's high-grade nature and the presence of intra-abdominal dissemination.
A review of the literature revealed 8 reported cases of primary gastric UPS (13-18). Based on an analysis of the 9 reported cases of primary gastric UPS, including ours, the mean age of the patients was 68 years (range: $42-79$ years), and the male-to-female ratio was 8:1 (Table I). The tumors measured between 3.5 and $12.0 \mathrm{~cm}$ in maximum diameter. As for the location of the sarcoma, it was located on the gastric body in 5 cases. There were no cases in which neoadjuvant or adjuvant chemotherapy was administered.

This was a very significant case, in that we were able to control a high-grade primary gastric sarcoma using surgical resection alone. The administration of adjuvant or neoadjuvant chemotherapy has been widely adopted as a treatment 


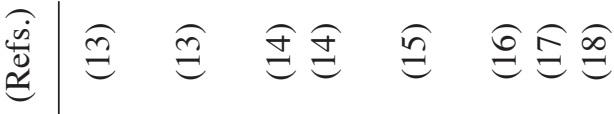

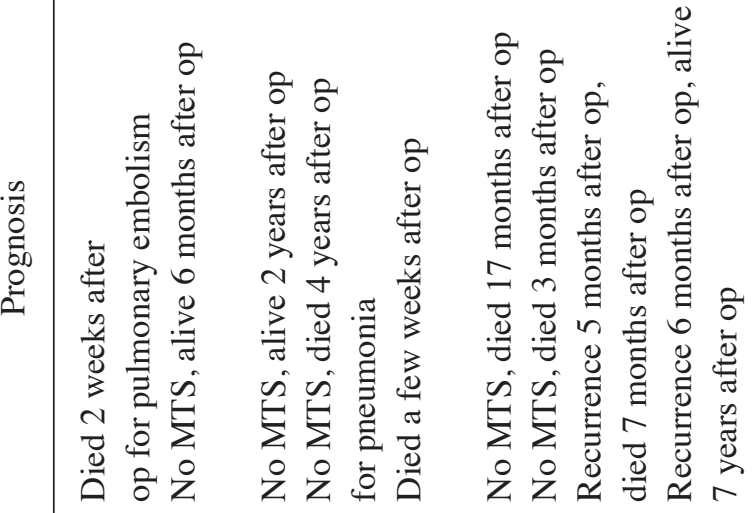

密

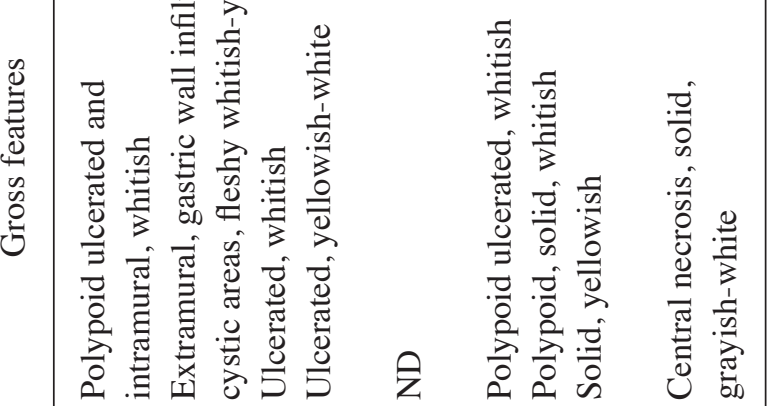

䓂

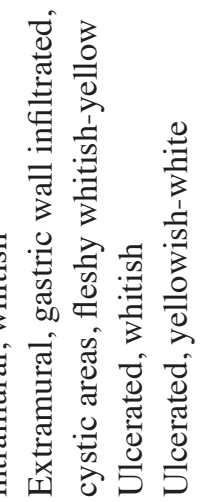

产

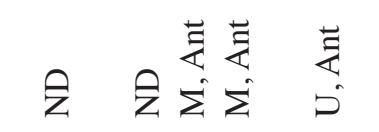

里

.

ֻृ

.

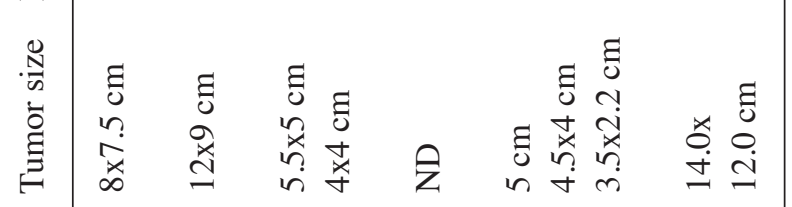

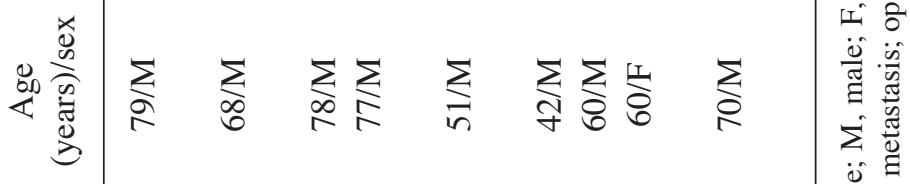

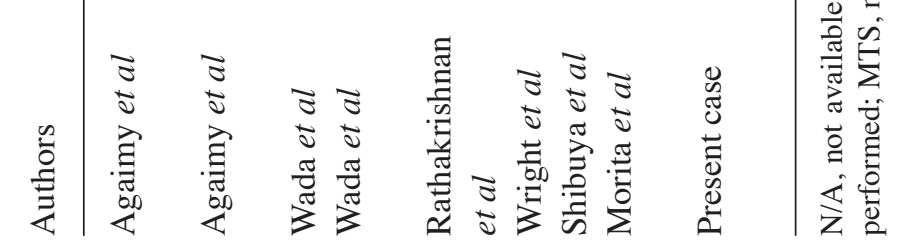


strategy for soft tissue sarcomas; however, there is no standard chemotherapy regimen for abdominal UPS, and we decided not to administer chemotherapy in the present case based on the low response rate of such tumors and the potential complications of chemotherapy $(19,20)$. According to the National Comprehensive Cancer Network soft tissue sarcoma guidelines, complete surgical resection with appropriate negative margins is the standard primary treatment for UPS (21). In spite of smaller surgical margin of gastric sarcoma than soft tissue sarcoma located at upper limbs or lower limbs, we were able to control this tumor via resection alone.

To the best of our knowledge, this is the first report about a case of primary gastric UPS involving positive peritoneal washing cytology results. Although the patient's good clinical course is difficult to explain based on the fact that the tumor was a high-grade sarcoma and peritoneal washing cytology produced positive results, we speculate that abdominal sarcomas like UPS do not have an invasive nature despite their high histological grade, which is unusual for this type of tumor. In the current case, continuous clinical observation for recurrence or metastasis will be necessary in case the tumor becomes more malignant.

In conclusion, this case deserves special mention, as it involved a high-grade sarcoma that was not treated with chemotherapy, and yet the patient exhibited a good prognosis. Clinicians should be aware that some sarcomas might follow a favorable course.

\section{Acknowledgements}

Not applicable.

\section{Funding}

No funding was received.

\section{Availability of data and materials}

The datasets obtained and/or analyzed during the current study are available from the corresponding author on reasonable request.

\section{Authors' contributions}

YO, HC and TM analyzed patient data and wrote the manuscript. RO, HO and RT collected the data and critically revised the manuscript.

\section{Ethics approval and consent to participate}

This case report was approved by the institutional review board, and written informed consent was obtained from the patient.

\section{Patient consent for publication}

Written consent for publication was obtained from the patient.

\section{References}

1. Fletcher CDM, Bridge JA, Hogendoorn P and Mertens F: WHO classification of tumours of soft tissue and bone. 4th edition. IARC Press, Lyon, pp120-122, 2013.

2. O'Brien JE and Stout AP: Malignant fibrous xanthomas. Cancer 17: 1445-1455, 1964.

3. Sano T and Aiko T: New Japanese classifications and treatment guidelines for gastric cancer: Revision concepts and major revised points. Gastric Cancer 14: 97-100, 2011.

4. Soufi M, Errougani A and Chekkof RM: Primary gastric leiomyosarcoma in young revealed by a massive hematemesis. J Gastrointest Cancer 40: 69-72, 2009.

5. Kabashima A, Kimura K, Sanefuji K, Masunari S, Haraoka S and Maekawa S: A case of primary gastric undifferentiated high-grade pleomorphic sarcoma diagnosed with chief complaint of fever: A case report and literature review. Surg Case Rep 3: 41, 2017.

6 . Welch JP: Smooth muscle tumors of the stomach. Am J Surg 130: 279-285, 1975.

7. Farid M, Demicco EG, Garcia R, Ahn L, Merola PR, Cioffi A and Maki RG: Malignant peripheral nerve sheath tumors. Oncologist 19: 193-201, 2014.

8. Virgilio E, Mercantini P, Tarantino G, Socciarelli F, Pilozzi E, Uccini S, Caterino S and Ziparo V: Gastrointestinal stromal tumors with de novo anaplastic dedifferentiation: Considerations on a little-known neoplastic metamorphosis. Int J Surg Pathol 22: 385,2014

9. McGovern Y, Zhou CD and Jones RL: Systemic therapy in metastatic or unresectable well-differentiated/dedifferentiated liposarcoma. Front Oncol 7: 292, 2017.

10. Villanueva A, Pérez C, Sabaté JM, Llauger J and Monill JM: CT manifestations of peritoneal leiomyosarcomatosis. Eur J Radiol 17: 166-169, 1993.

11. Giganti F, Orsenigo E, Esposito A, Chiari D, Salerno A, Ambrosi A Albarello L, Mazza E, Staudacher C, Del Maschio A, et al: Prognostic role of diffusion weighted MR imaging for resectable. Gastric cancer. Radiology 276: 444-452, 2015.

12. Italiano A, Delva F, Mathoulin-Pelissier S, Le Cesne A, Bonvalot S, Terrier P, Trassard M, Michels JJ, Blay JY, Coindre JM and Bui B: Effect of adjuvant chemotherapy on survival in FNCLCC grade 3 soft tissue sarcomas: A multivariate analysis of the French Sarcoma Group database. Ann Oncol 21: 2436-2441, 2010.

13. Agaimy A, Gaumann A, Schroeder J, Dietmaier W, Hartmann A, Hofstaedter F, Wünsch PH and Mentzel T: Primary and metastatic high-grade pleomorphic sarcoma/malignant fibrous histiocytoma of the gastrointestinal tract: An approach to the differential diagnosis in a series of five cases with emphasis on myofibroblastic differentiation. Virchows Arch 451: 949-957, 2007.

14. Wada Y, Matsushita T, Sarumaru S, Ryo J, Isobe H, Satoh B, Kanaya S, Katayama T and Ohtoshi M: Malignant fibrous histiocytoma of the stomach: Report of two cases. Surg Today 28: 296-300, 1998

15. Rathakrishnan V, Arianayagam S and Kumar G: Primary malignant fibrous histiocytoma of the stomach: (A case report). Australas Radiol 33: 302-304, 1989.

16. Wright JR Jr, Kyriakos M and DeSchryver-Kecsemeti K: Malignant fibrous histiocytoma of the stomach. A report and review of malignant fibrohistiocytic tumors of the alimentary tract. Arch Pathol Lab Med 112: 251-258, 1988.

17. Shibuya H, Azumi N, Onda Y and Abe F: Multiple primary malignant fibrous histiocytoma of the stomach and small intestine. Acta Pathol Jpn 35: 157-164, 1985.

18. Morita T, Kato T, Furukawa K and Itoh Y: A case report of malignant fibrous histiocytoma in the regions of stomach and gall bladder. J Jpn Soc Clin Cytol 23: 425-429, 1984.

19. Pasquali S and Gronchi A: Neoadjuvant chemotherapy in soft tissue sarcomas: Latest evidence and clinical implications. Ther Adv Med Oncol 9: 415-429, 2017.

20. Guo J, Cui Q, Liu C, Sui J, Jiang N, Zhou J, Li D and Zeng Y: Clinical report on transarterial neoadjuvant chemotherapy of malignant fibrous histiocytoma in soft tissue. Clin Transl Oncol 15: 370-375, 2013.

21. NCCN Guidelines for Patients. Soft Tissue Sarcoma. Version 1.2014. https://www.nccn.org/patients/guidelines/sarcoma. Accessed April 12, 2018.

\section{Competing interests}

The authors declare that they have no competing interests. 